\title{
Health Promotion: Looking Back... Moving Forward
}

\author{
ANuPam Sachdeva \\ National President, Indian Academy of Pediatrics, 2017 \\ anupamace@yahoo.co.in
}

$\mathrm{H}$ ealth promotion is the process of enabling people to increase control over, and to improve, their health. It was 30 years ago that World Health Organization (WHO) had conducted its first conference on health promotion. At its conclusion, they adopted 'Ottawa charter for health promotion'. It was a hinge between past and future as it pooled the principles and ideas that were evolving around five major strategies and expressed a vision of the future.

The practice of public health has been dynamic in India. The major public health problems like malaria, tuberculosis, leprosy, high maternal and child mortality and human immunodeficiency virus (HIV) have been addressed well, leading to decrease in the mortality rates [1]. However, there are multiple problems yet to be focussed on. As people are divided into various social strata as per their income, occupation, education, race or ethnicity, the inequalities in health are also seen. To meet the formidable challenges, there is an urgent call for revitalizing primary health care based on the principles outlined at Alma-Ata in 1978: Universal access and coverage, equity, community participation in defining and implementing health agendas, and intersectoral approaches to health. Attempts to achieve "Health for All" have been carried forward in the form of "Millenium Development Goals" [1]. However, the role of government is crucial for addressing these challenges and achieving health equity. The Ministry of Health and Family Welfare (MOHFW) plays a key role in guiding India's public health system. Contribution to health of a population derives from systems outside the formal health care system, and this potential of intersectoral contributions to the health of communities is increasingly recognized worldwide. Thus, the role of government in influencing population health is not limited within the health sector but also by various sectors outside the health systems [1].

\section{Health System Strengthening}

Important issues that the health systems must confront are: lack of financial and material resources, health workforce issues and the challenge of implementing health policies in a pluralistic environment [1]. The National Rural Health Mission (NRHM) launched by the Government of India is a leap forward in establishing effective integration and convergence of health services, and affecting architectural correction in the health care delivery system in India. Mechanisms to monitor epidemiological challenges like mental health, occupational health and other environment risks are yet to be put in place.

\section{Conclusion}

Promotion of multisectoral approach within the health system and with other ministries is seen as an important measure for effective implementation. Strengthening communication is one of the key requisites for ensuring multisectoral coordination [2]. Social determinants of health and economic issues must be dealt with a consensus on ethical principles - universalism, justice, dignity, security and human rights.

It is true that a lot has been achieved in the past. The milestones in the history of public health that have had a telling effect on millions of lives - launch of Expanded Program of Immunization in 1974, Primary Health Care enunciated at Alma-Ata in 1978, eradication of smallpox in 1979, launch of polio eradication in 1988, and COTPA Act of 2005 , etc. It was a glorious past, but the future of a healthy India lies in mainstreaming the public health agenda in the framework of sustainable development.

\section{REFERENCES}

1. Lakshminarayanan S. Role of government in current health: Current scenario in India and future scope. J Family Community Med. 2011;18:26-30.

2. Salunke S, Lal DK. Multisectoral approach for promoting public health. Indian J Public Health. 2017;61:163-8. 